\title{
Working with People
}

\section{The Elements}

1 Teamwork

2 Interviewing

3 Negotiation

4 Consultation
Guide Prior Time Elements * 26-29 hrs

17-24 hrs

8-10 hrs

13-15 hrs

* $\quad$ All Elements are designed to 'stand alone' and can be tackled in any sequence. 